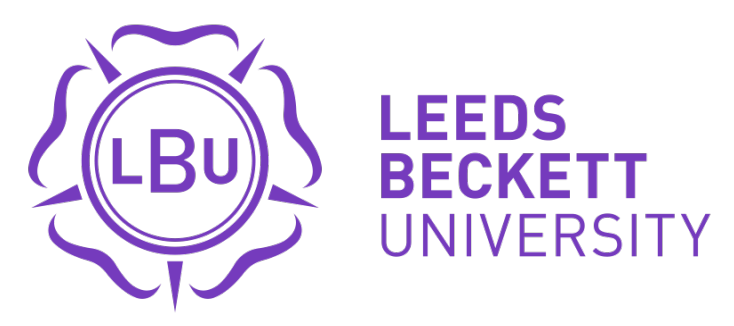

Citation:

Simmons, S and Estes, Z (2008) Individual differences in the perception of similarity and difference. Cognition, 108 (3). 781 - 795. ISSN 0010-0277 DOI: https://doi.org/10.1016/j.cognition.2008.07.003

Link to Leeds Beckett Repository record:

https://eprints.leedsbeckett.ac.uk/id/eprint/1007/

Document Version:

Article (Accepted Version)

The aim of the Leeds Beckett Repository is to provide open access to our research, as required by funder policies and permitted by publishers and copyright law.

The Leeds Beckett repository holds a wide range of publications, each of which has been checked for copyright and the relevant embargo period has been applied by the Research Services team.

We operate on a standard take-down policy. If you are the author or publisher of an output and you would like it removed from the repository, please contact us and we will investigate on a case-by-case basis.

Each thesis in the repository has been cleared where necessary by the author for third party copyright. If you would like a thesis to be removed from the repository or believe there is an issue with copyright, please contact us on openaccess@leedsbeckett.ac.uk and we will investigate on a case-by-case basis. 


\begin{abstract}
Thematically related concepts like coffee \& milk are judged to be more similar than thematically unrelated concepts like coffee \& lemonade. We investigated whether thematic relations exert a small effect that occurs consistently across participants (i.e., a generalized model), or a large effect that occurs inconsistently across participants (i.e., an individualized model). We also examined whether difference judgments mirrored similarity or whether these judgments were, in fact, non-inverse. Five studies demonstrated the necessity of an individualized model for both perceived similarity and difference, and additionally provided evidence that thematic relations affect similarity more than difference. Results suggest that models of similarity and difference must be attuned to large and consistent individual variability in the weighting of thematic relations.
\end{abstract}

KEYWORDS: dual process model; individual differences; non-inversion; perceived difference; perceived similarity; thematic relations. 
Similarity and difference are fundamental to cognition. They determine, in large part, the recognition of familiar objects and the categorization of novel objects, and they drive inferences about an object's features and its predicted behavior in a novel context. Essentially, similarity and difference have been implicated in nearly every cognitive process from perceptual classification to economic decision-making. Given that similarity and difference influence so many other cognitive processes, then, it is crucial to determine what factors influence the perceptions of similarity and difference themselves.

Some potentially important factors remain underspecified in contemporary models of similarity and difference. For example, perceived similarity and difference may exhibit large individual differences (Simmons \& Estes, 2008), but current models fail to account for this presumed variability. Moreover, the relationship between similarity and difference appears to be surprisingly complex (Medin, Goldstone, and Gentner, 1990), and current models do not adequately explain this relationship. Below we report five studies that investigate directly these currently unresolved issues. We first review the major models of semantic similarity, with particular emphasis on the dual process model (Bassok \& Medin, 1997; Estes, 2003; Simmons \& Estes, 2008; Wisniewski \& Bassok, 1999), and then we summarize the literature on the relationship between similarity and difference.

\section{Models of Similarity}

In general, similarity can be described in terms of three types of information: features, structural relations, and thematic relations. Of these, features make the most intuitive contribution - the more features that two things have in common, the more similar they are. This intuition is formalized in the contrast model (Tversky, 1977), which construes the similarity of two concepts as a function of their common features weighed against their distinctive features. Similarity increases as the number of commonalities increases or the number of differences decreases. For example, a bluebird and a crow are perceived to be 
similar because their many common features (beaks, wings, etc) outweigh their few distinctive features (size, color). And if the bluebird were to grow black feathers, then its similarity to a crow would increase still further (Gati \& Tversky, 1984).

However, features alone are not sufficient for determining similarity. To understand why, consider the following example from Medin, Goldstone, and Gentner (1993): "Person 1 has on a black and white striped shirt and red and green checkered pants, and Person 2 has on a black and white checkered shirt and red and green striped pants" (p. 260). How similar are Person 1 and Person 2? Both wear one striped item and one checkered item, and each wears one black and white item and one red and green item. So by a simple feature matching model such as the contrast model, these two people are wearing maximally similar costumes. Such a model fails to capture the relationship between the pattern and the coloring of each garment.

The structural alignment model (Gentner \& A. B. Markman, 1994, 1997; A. B. Markman, 1996; A. B. Markman \& Gentner, 1993a, 1993b) provides a solution to this problem. According to this model, one's concepts are structured representations that encode features as well as the relationships among features (i.e., structural relations). Judging similarity involves bringing two representations into alignment, so as to maximize conceptual overlap. Returning to the above example, the representations must be aligned so that one shirt is compared directly to the other shirt, and likewise for the pants. This process highlights the similarity between their conceptual structure (i.e., both are wearing shirts and pants), and it draws attention to differences related to this structure (i.e., the two shirts have different patterns, as do the pants). Thus, structural relations are a necessary and important component of any model of similarity.

Thematic relations also have a significant, albeit smaller, influence on perceived similarity (see Simmons \& Estes, 2008). The critical contrast between structural relations and thematic relations is that whereas structural relations occur within a single stimulus, thematic 
relations occur between stimuli. To illustrate, a flower is connected to its stem in the same way that an eraser is attached to a pencil, thereby constituting a similarity of structural relations. But flowers and pencils are not thematically related. In contrast, flowers and bees are thematically related, but share few if any structural relations. An extensive body of research indicates that structural relations guide the comparison process, which in turn largely determines the perception of similarity and difference (for review see Gentner \& A. B. Markman, 1997). Of greater interest for the present purposes, however, is a growing body of evidence suggesting that thematic relations also influence perceived similarity and difference. Indeed, the importance of thematic relations to similarity judgments has been demonstrated across a variety of stimuli and tasks. Bassok and Medin (1997) found that when judging the similarity of two sentences containing the same nouns (e.g., The carpenter fixed the chair \& The carpenter sat on the chair), participants justified their ratings by integrating the sentences into a common scenario (e.g., "[T] he fixed it well"). Thus, participants justified similarity not by comparing structural relations (e.g., both sentences are about carpenters), but by inventing a novel thematic relation to link the actions in each sentence. The most direct evidence for a thematic influence on similarity is the finding that thematically related concepts are judged to be more similar than thematically unrelated concepts. For example, Wisniewski and Bassok (1999) demonstrated that participants rated apple pie \& ice cream as more similar than apple pie \& jello, presumably because of the thematic link between apple pie and ice cream (i.e., the two often are eaten together). Estes (2003) had one group of participants simply rate the similarity of concept pairs such as pancake \& spatula, whereas another group of participants thematically integrated the concepts prior to rating their similarity. The same two concepts were judged more similar by participants who had integrated them than by participants who had not. More recently, Jones and Love (2007) explicitly stated a relation between two concepts (e.g., The 
collie herds the sheep) before soliciting ratings of their similarity. Their results corroborated the observation that a thematic relation between two concepts increases the perceived similarity of those concepts.

The structural alignment model fails to explain this influence of thematic relations on perceived similarity and difference. For example, from the perspective of alignment theory, assessing the similarity of apple pie and ice cream requires aligning the two representations to apprehend commonalties (both are desserts, both are sweet) and differences related to conceptual overlap (ice cream is colder than apple pie). Structural alignment accounts for the relations between the features of a single stimulus, but it often underestimates perceived similarity because it fails to account for the relation between two stimuli. In fact, Gentner and Brem (1999; see also Gentner \& Gunn, 2001) reject thematic relations as a legitimate source of similarity. In their view, similarity is synonymous with comparison (i.e., structural alignment); therefore, any other process that influences a similarity task (e.g., thematic integration) is considered illegitimate.

Importantly, thematic relations cannot be represented as commonalities of the form "Both X and Y participate in scenario Z." Gentner and Gunn (2001) presented pairs of concepts and instructed participants to list either thematic relations between the items or commonalities. They found that the two tasks had different effects on the subsequent processing of those stimuli: Whereas listing thematic relations inhibited the detection of differences between concepts, listing commonalities facilitated the detection of differences. Critically, if a thematic relation between two concepts was merely a commonality between them, then listing thematic relations and listing commonalities should have elicited similar effects. Thus, thematic relations are not represented as common features and cannot be accounted for by a single process model (see also Simmons \& Estes, 2008). 
The dual process model (Bassok \& Medin, 1997; Estes, 2003; Wisniewski \& Bassok, 1999) accommodates the influence of thematic relations simply by allowing both comparison (i.e., structural alignment) and integration to affect similarity. According to the model, the relative influences of the comparison and integration processes will depend on the relationship between the items: Comparison is more heavily weighted for structurally alignable items (i.e., those with similar conceptual structure), whereas integration is more heavily weighted for thematically related items. Furthermore, the relative dominance of a given process can also be mediated by the task itself: Structural alignment should dominate similarity judgments, and thematic integration should dominate relational judgments. Thus, the dual process model predicts that stimulus and task factors interact to determine which processing mechanism (i.e., alignment or integration) will dominate judgment. A further question of interest — which will serve as the focus of the present investigation - is whether the relative dominance of these distinct processing mechanisms also varies systematically across participants.

\section{Individual Differences in Similarity}

The dual process model tacitly assumes a generalized effect of thematic relations on judgments of similarity. That is, most or all participants are predicted to rate items like milk \& coffee to be slightly more similar than pairs like milk \& lemonade, because the former pair is thematically related but the latter pair is not. By this standard version of the dual process model, which we will refer to as a generalized model, each participant exhibits a thematic effect. As an alternative, we note that the small thematic effect observed in prior tasks might instead be attributable to substantial individual differences in the perception of similarity. By an individualized model, some participants assign milk \& coffee a much higher similarity rating than milk \& lemonade, while other participants assign the two pairs roughly equal ratings. Put differently, there may be a large thematic effect for a subset of participants only, 
with little or no thematic effect for other participants. The relatively small thematic effect observed in prior studies may result from averaging across these two groups of participants.

If the generalized model is correct, then certainly much remains to be specified in the dual process model (e.g., how thematic integration occurs), but the basic model delineated by Wisniewski and Bassok (1999) would provide a sound framework for further development. In contrast, if the individualized model were correct, then models of similarity would require more substantial revision. In particular, models of similarity may require the addition of an individual parameter, and in fact, this parameter may ultimately prove more important than either the stimulus parameter or the task parameter. The purpose of the present investigation, therefore, was to discriminate between the generalized and individualized versions of the dual process model.

Preliminary evidence from research on categorization supports the individualized model. A traditional assumption about category learning is that adults form taxonomic (comparison-based) rather than thematic (integration-based) categories. Lin and Murphy (2001; see also Murphy, 2001) found across several studies that many participants consistently categorized a base concept (camel) with a taxonomically related alternative (antelope) rather than with a thematically related alternative (desert). But a substantial number of other participants consistently categorized the base concepts with the thematically related alternative instead. To the extent that categorization is based on similarity, it is likely that comparable individual differences will be observed in similarity judgments as well. Indeed, we have found elsewhere that some participants exhibit a systematic dissociation of similarity and categorization judgments, while other participants exhibit no such dissociation (Hampton, Estes, \& Simmons, 2007). Thus, research on categorization suggests that different individuals may perceive similarity in very different ways. 
Some prior research on similarity indicates that individual differences in similarity are associated with thematic integration. Gentner and Brem (1999) describe a screening task in which 702 participants were presented triads consisting of a base, a taxonomic alternative, and a thematic alternative (cf. Lin \& Murphy, 2001). Participants were instructed to choose the alternative that was most similar to the base. Of Gentner and Brem's sample, $41 \%$ of participants exhibited no systematic preference for either alternative (i.e., taxonomic or thematic). However, $48 \%$ consistently chose the taxonomic alternative, while $11 \%$ consistently chose the thematic alternative (see also Simmons \& Estes, 2008, described below). This study indicates systematic individual differences in the perception of similarity.

\section{Non-Inversion of Similarity and Difference}

The simplest model of similarity and difference would hold that these factors are complementary, or inverse. That is, as similarity increases, difference decreases to the same extent. For instance, if cats and dogs have a similarity rating of 6 on a 1-to-7 scale, then they ought to have a difference rating of 2 . However, in certain cases perceived similarity is not inversely related to perceived difference. Medin et al. (1990) demonstrated a non-inversion between similarity and difference using configurations of geometric shapes in a triad task. For a given target item (e.g., a black square above a white circle) one response option matched in relation information but mismatched in attribute information (e.g., a white square above a white circle), whereas the other matched in attribute information but mismatched in relation information (e.g., a black square next to a white circle). When asked to pick the alternative that was more similar to the target, participants usually chose the relation match. However, participants also usually chose the relation match when the task was to pick the alternative that was more different from the target. That is, participants chose the same items as both more similar and more different. Estes and Hasson (2004) found a similar pattern of results. In their experiment, participants preferred the relation match in the similarity condition, but 
showed no preference in difference. Bassok and Medin (1997) demonstrated non-inversion in a sentence comparison task. Participants judged a base sentence (The carpenter fixed the chair) as more similar to a relational match (The electrician fixed the radio) than to an attribute match (The carpenter sat in the chair). But once again, participants demonstrated no preference in difference judgments. In each of these studies, the non-inversion was attributable to a greater relative weighting of structural relations in similarity than in difference.

Simmons and Estes (2008) tested whether thematic relations might also induce a noninversion of similarity and difference. We solicited from participants both similarity ratings and difference ratings (between-participants) for the same pairs of thematically related and unrelated concepts. Results corroborated the influence of thematic relations on perceived similarity (Wisniewski \& Bassok, 1999). But more importantly, this thematic effect was significantly attenuated in difference ratings, thereby producing a non-inversion. In Experiment 1, for instance, thematic relatedness accounted for $54 \%$ of the variance in similarity ratings, but only $34 \%$ of the variance in difference ratings. In another experiment we explicitly instructed participants to base their ratings on features alone, and participants generally were able to discount thematic relations from their ratings. This exclusion of thematic relations substantially reduced the non-inversion. In a final experiment we administered a similarity triad task (cf. Gentner \& Brem, 1999; Lin \& Murphy, 2001) prior to the similarity or difference rating task. Consistent with previous studies, the triad task revealed substantial individual variability in perceived similarity. More interesting, though, was the finding that participants' preference for thematic alternatives in the triad task better predicted their subsequent similarity ratings than their difference ratings. So, again thematic relations were more closely associated with perceived similarity than with perceived difference. Even more strikingly, participants who preferred thematic choices exhibited a non- 
inversion of similarity and difference, whereas participants who preferred taxonomic choices did not exhibit the non-inversion. These experiments provide initial evidence that thematic relations are more heavily weighted in similarity judgments than in difference judgments, and that this effect is observed only in a subset of participants.

In summary, although the generalized dual process model (e.g., Wisniewski \& Bassok, 1999) currently offers the only account of thematic relations, more recent evidence appears to support an individualized model instead. Below we report a series of studies that examine in greater detail the presumed individual variability of perceived similarity and difference.

\section{Experiment 1a}

The purpose of Experiment 1a was to provide an initial test of the generalized and individualized models. The experiment also tested whether a preference for thematic choices is related to the non-inversion of similarity and difference, as suggested by Simmons and Estes (2008). Using the triad choice task (Gentner \& Brem, 1999; E. M. Markman \& Hutchinson, 1984; see also Lin \& Murphy, 2001), participants chose whether a taxonomic alternative or a thematic alternative was more similar to (or more different from) a base stimulus. For instance, the base stimulus bee was presented with alternative choices fly and honey, and participants chose the alternative that was either more similar to or more different from the base (between-participants). The generalized model (Bassok \& Medin, 1997; Estes, 2003; Wisniewski \& Bassok, 1999) assumes between-participant agreement. That is, the proportion of thematic choices should be relatively constant across participants. Alternatively, the individualized model assumes that the dominance of comparison or integration is largely determined by the individual. Thus, the individualized model predicts between-participant disagreement; many thematic respondents and many taxonomic respondents should be observed. Finally, if thematic relations are more relevant to similarity than to difference, as 
suggested by the results of Simmons and Estes (2008), then thematic choices should be more common in similarity than in difference judgments.

Method

Participants. Participants in all experiments were undergraduates at the University of Georgia, all were native English speakers, and all received course credit for participation. In Experiment 1a, 68 participants were randomly assigned to a Similarity condition $(n=35)$ or a Difference condition $(\mathrm{n}=33)$.

Materials \& Procedure. Thirty triads were sampled from Lin and Murphy (2001, Table 1, items 1-30). Each triad consisted of a base (pencil), a thematically related option (eraser), and a taxonomically related option (pen). On each trial the base appeared in the center of a computer display, above the response options, which appeared left and right of center on the same line. Participants in the Similarity condition were instructed to "Pick the response option that is most similar to [base]" by pressing the $<1>$ or the $<2>$ key. The Difference condition was identical except that participants were instructed to choose the option "most different from" the base. Assignment of taxonomic and thematic options to the left and right positions was counterbalanced across two lists. Each participant judged all 30 triads, which appeared in random order.

Results

Agreement was tested across participants (Lin \& Murphy, 2001), whereas noninversion was tested across items (Medin et al., 1990). These analyses are reported separately below.

Agreement. For each participant we calculated the proportion of trials on which the thematic option was chosen (thematic proportion). Participants were classified into three groups on the basis of binomial probability. Given 30 triads, any participant whose thematic proportion was between .31 and .69 (inclusive) exhibited no significant preference for either 
taxonomic or thematic options. These participants constituted a "Mixed" response group. Participants who exhibited a significant preference for taxonomic options (i.e., thematic proportion $<.31$, binomial $\mathrm{p}<.05$ ) were dubbed the "Taxonomic" group, and those who significantly preferred the thematic option (i.e., thematic proportion $>.69$, binomial $p<.05$ ) constituted a "Thematic" group.

Table 1 and Figure 1 illustrate the distribution of participants among these groups. Participants tended to exhibit a clear preference for either taxonomic or thematic choices; only $23 \%$ and $21 \%$ of participants in the similarity and difference conditions (respectively) chose the taxonomic and thematic options in roughly equal measure. Whereas $31 \%$ of participants consistently chose the taxonomic option as more similar to the base, $46 \%$ reliably chose the thematic option as more similar. The same pattern of contrasting choices was observed in the difference condition. Whereas $39 \%$ systematically chose the taxonomic option as more different from the base, another 39\% regularly selected the thematic option.

The prevalence of thematic choices among some participants could reflect a task demand to respond consistently across trials. For example, a participant who chose the thematic match on the first trial might have selected thematic matches for the remainder of the experiment in order to appear consistent (see Lin \& Murphy, 2001, p. 10-11). If so, then participants who judged a triad with a high thematic proportion on their first trial should be more likely to have a high overall thematic proportion. In other words, participants' overall thematic proportions should correlate with the average thematic proportion of the item they judged on the first trial. However, this correlation did not approach significance in either similarity $[r=.05, p=.76]$ or difference judgments $[r=-.20, p=.26]$. Thus, the prevalence of thematic choices was not attributable to a consistency bias.

Non-inversion. For each triad we calculated the proportion of thematic choices in the similarity condition and in the difference condition separately. Figure 2 plots these 
proportions. The negative diagonal represents the theoretical inverse relationship between similarity and difference. If similarity and difference were inverses, then the 30 triads should be distributed evenly around the negative diagonal, and the overall proportion of thematic choices (collapsed across the similarity and difference conditions) should be .50 . To illustrate, if a given triad elicited $25 \%$ thematic choices in the similarity condition, then complementarity would predict $75 \%$ thematic choices in the difference condition, so that averaging across the similarity and difference conditions would yield $50 \%$ thematic choices. Any deviation from this complementarity (i.e., thematic proportion $=.50$ ) would constitute a non-inversion of similarity and difference (Medin et al., 1990).

As evident in Figure 2, the similarity and difference choices were indeed negatively correlated, although the strength of this relationship was surprisingly moderate $[\mathrm{r}(30)=-.53$, $\mathrm{p}<.01]$. More importantly, the stimuli tended to occur above the diagonal, with fewer observations below it. This property is confirmed by the overall proportion of thematic choices $(\mathrm{M}=.52, \mathrm{SE}<.01)$, which deviated significantly from complementarity, $\mathrm{t}(29)=$ $2.18, p<.05$. Thus, similarity and difference were non-inverse. More specifically, the proportion of thematic choices was above chance for similarity $[\mathrm{M}=.54, \mathrm{SE}=.02 ; \mathrm{t}(29)=$ $2.34, p<.05]$, but was at chance for difference $[\mathrm{M}=.49, \mathrm{SE}=.02 ; \mathrm{p}=.69]$. As suggested by Simmons and Estes (2008), thematic choices dominated in similarity, but thematic and taxonomic choices were equiprobable in difference.

\section{Discussion}

Distinct groups of participants exhibited similarity judgments that strongly contrasted one another, and the same occurred for difference judgments as well. These results cannot be explained by the contrast model (Navarro \& Lee, 2004; Tversky, 1977) or by structural alignment (e.g., Gentner \& A. B. Markman, 1997). If similarity and difference were based on comparison only, then thematic choices should occur rarely (if ever). Thus, these models fail 
to explain the prevalence — indeed, the dominance — of thematic choices. The generalized model, which includes both comparison and thematic integration (Bassok \& Medin, 1997; Estes, 2003; Wisniewski \& Bassok, 1999), provides only a partial explanation. Although this model can account for a consistent amount of thematic responding across participants, it does not explain the systematic individual differences in similarity judgments. Only the individualized model can explain these results. For some participants (i.e., the Taxonomic group in similarity and the Thematic group in difference), similarity and difference do appear to favor feature comparison. But for others (i.e., the Thematic group in similarity and the Taxonomic group in difference), thematic relatedness is clearly more central to similarity and difference. And yet a third group (i.e., Mixed) appear to weight the feature comparison and thematic integration processes more equally. Notably, however, this mixed responding was relatively rare. So evidently, similarity and difference both entail dual processes of comparison and integration, but the relative weightings of these two factors vary dramatically across participants.

Furthermore, between-participant disagreement produced a non-inversion of similarity and difference. That is, thematic choices were more common than taxonomic choices in the similarity condition but thematic and taxonomic choices were equiprobable in the difference condition. So the thematic dominance in similarity was not complemented by a taxonomic dominance in difference, and hence a non-inversion of similarity and difference occurred (see Figure 2).

One could argue that the non-inversion was simply the product of variability across samples (e.g., we happened to sample more Thematic participants in the similarity condition). We believe that this is unlikely. Previous demonstrations of non-inversion also manipulated judgment between participants (Bassok \& Medin, 1997; Estes \& Hasson, 2004; Medin et al., 1990; Simmons \& Estes, 2008), and in each of those experiments, relational matches tended 
to be selected as both more similar to and more different from a base concept. If the effect were attributable to random sampling error, then some of these studies would be expected to exhibit the opposite effect - that is, a tendency to select attribute matches as both more similar and more different. Nonetheless, this sampling explanation of non-inversion is investigated more directly in Experiment 2.

\section{Experiment $1 b$}

The previous experiment demonstrated between-participant disagreement in similarity and difference judgments. However, similarity judgments are highly sensitive to a variety of task factors (Barsalou, 1982; Beck, 1966; Gentner \& Brem, 1999), including the precise wording of the similarity question (e.g., Goldstone, 1994). In particular, Jones and Love (2007) found that participants are less likely to respond thematically when asked about how like items are, rather than how similar they are. Therefore, in order to test whether the prevalence of thematic responding might have resulted from a misconstrual of the term "similarity", in this study we asked participants to choose the alternative that was more "like" the base. If the occurrence of thematic responding were contingent on this minor wording manipulation, it would undermine the supposition that thematic responding reflects a deeper conceptual preference. In contrast, between-participant disagreement under this condition would demonstrate the robustness of individual differences in the perception of similarity. Method

Thirty-five undergraduates participated. The procedure was identical to the similarity condition in Experiment 1a, except that for each item the word "like" was substituted for "similar to."

Results

Despite substituting "like" for "similar to", between-participant disagreement persisted (see Table 1). Nearly a third (31\%) of participants was classified as Taxonomic, 
while a full $57 \%$ of participants were classified as Thematic. Only $12 \%$ fell into the Mixed category. As in Experiment 1a, the preference for thematic matches was above chance $[\mathrm{M}=$ $.60, \mathrm{SE}=.01 ; \mathrm{t}(29)=8.71, \mathrm{p}<.001]$. In fact, this distribution of participants among the three response groups was statistically indistinguishable from the distribution in the similarity condition of Experiment 1a (left half of Figure 1) $\left[\chi^{2}(2)=1.78, p=.41\right]$. In other words, "similar to" and "like" elicited analogous patterns of responding.

\section{Discussion}

It is apparent from these results that the same between-participant disagreement obtained even though the task was designed to discourage thematic responses (Jones \& Love, 2007). This suggests a deeper conceptual basis for disagreement that cannot be dismissed as a misinterpretation of "similarity." Participants appear to have strikingly different beliefs about the nature of similarity. To some participants common features are more important, while to others the relations between concepts are the most critical. Results therefore necessitate an individualized model of perceived similarity.

\section{Experiment 2a}

The presence of individual differences in similarity and difference judgments provides an important theoretical constraint on models of similarity. Moreover, those models are constrained further by the non-inversion of similarity and difference. All prior demonstrations of non-inversion (including Experiment 1a) have elicited similarity and difference judgments between-participants, so it is unclear whether individual participants exhibit non-inversion or whether it occurs only at the group level. In other words, do participants simply always select the same type of option (thematic or taxonomic) regardless of the task (similarity or difference), or do individual participants treat similarity and difference judgments as inverses? Perhaps eliciting both similarity and difference judgments of the same items from the same participants would reveal a complementary relationship between the measures, thereby 
eliminating non-inversion. Presumably, if a participant judges pencil as more similar to eraser than to pen, that same participant would judge pencil as more different from pen than from eraser. If this is the case, then individual differences are relatively stable across judgments; a Thematic participant in similarity should become a Taxonomic participant in difference, and vice versa. To test this possibility, Experiment 2a was an exact replication of Experiment 1a, except that similarity and difference judgments were collected within-participants in two different phases of the experiment. If the same individuals were reliably to choose a given option as both more similar to and more different from its base, that would provide strong evidence of non-inversion. Furthermore, if the non-inversion is attributable to a greater reliance on thematic integration than on feature comparison, Thematic participants should be more likely than Taxonomic participants to exhibit non-inversion.

\section{Method}

Fifty-five undergraduates participated. The materials and procedure were identical to Experiment 1a, except that each participant judged both the similarity and the difference of all 30 triads. At the beginning of the session, participants were randomly assigned to complete either the similarity judgments or the difference judgments. Participants then completed several unrelated tasks (e.g., a mediated priming experiment), and after approximately 20-30 minutes they completed the other judgments. Thus the similarity and difference judgments were blocked, counterbalanced, and separated by approximately 20 minutes.

\section{Results}

Agreement. Participants were classified into response groups according to the criteria established in Experiment 1. To test for effects of presentation order (similarity first or difference first) we conducted a 2 (order) by 2 (judgment) ANOVA on thematic proportions with participants random. Because neither the main effect of order $[\mathrm{F}(1,53)=.54, \mathrm{p}=.47]$ 
nor the order by judgment interaction $[\mathrm{F}(1,53)=3.53, \mathrm{p}=.07]$ was significant, we collapsed across presentation order for the remaining analyses.

As in Experiment 1a, the distribution of participants' thematic proportions indicated between-participant disagreement in similarity and difference judgments (see Table 1 and Figure 3$)$. In the similarity condition, over half of participants $(55 \%)$ chose the taxonomic option most of the time, while over a third (38\%) chose the thematic option for most trials. Only $7 \%$ of participants had thematic proportions in the middle interval, indicating that most people demonstrated a preference for one or the other type of option. In the difference condition, $27 \%$ of participants tended to choose the taxonomic option, while $62 \%$ preferred the thematic option. The percentage of participants having thematic proportions in the middle interval was again relatively low (11\%), indicating that few people chose both option-types equally often. Thus, the present experiment replicated the between-participant disagreement in similarity and difference that was originally observed in the preceding experiment.

Non-inversion. The proportions of thematic choices in the similarity and difference conditions are plotted in Figure 4. Recall that if similarity and difference judgments were complementary, then the items should be distributed about the negative diagonal, and the overall proportion of thematic choices should be .50 . As in Experiment 1a, similarity and difference were indeed negatively correlated $[\mathrm{r}(30)=-.63, \mathrm{p}<.001]$, but the majority of items were distributed above the negative diagonal. Thus, the overall proportion of thematic choices $(\mathrm{M}=.54, \mathrm{SE}<.01)$ was significantly greater than $.50,[\mathrm{t}(29)=8.70, \mathrm{p}<.001]$. This result indicates that similarity and difference judgments were non-inverse again. In Experiment 1a the thematic option was preferred in similarity judgments $(\mathrm{M}=.54, \mathrm{SE}=.02)$, but the difference judgments exhibited no reliable preference $(\mathrm{M}=.49, \mathrm{SE}=.02)$. Here in Experiment $2 \mathrm{a}$ both similarity and difference judgments exhibited a reliable preference: Whereas the taxonomic option was preferred in similarity $[\mathrm{M}=.43, \mathrm{SE}=.01, \mathrm{t}(29)=-5.80, \mathrm{p}$ 
$<.001]$, the thematic option dominated in difference $[\mathrm{M}=.66, \mathrm{SE}=.01, \mathrm{t}(29)=15.34, \mathrm{p}<$ $.001]$.

Across participants, the same option was selected as both more similar and more different on $33 \%$ of trials. The vast majority of these individual cases of non-inversion occurred with thematic options: The thematic alternative was chosen as both more similar and more different on $24 \%$ of trials, while the taxonomic alternative was chosen in both judgments only $9 \%$ of the time. This finding explains why the overall thematic proportion was greater than .50 (see above).

Table 2 presents the distribution of participants among the three response group in each of the judgment tasks. If similarity and difference were inverse, then a participant who exhibited a taxonomic preference in similarity should exhibit a thematic preference in difference. Likewise, one who is Thematic in similarity should be Taxonomic in difference, and a Mixed participant should remain so across judgments. These groups of participants, who exhibited an inverse relationship between their similarity choices and their difference choices, appear in Table 2 as the positive diagonal (in bold font). As evident in the table, the most frequent pattern was of taxonomic similarity choices and thematic difference choices (45\%). But perhaps more interestingly, $20 \%$ of participants showed a thematic preference in similarity and a taxonomic preference in difference. A further $4 \%$ of participants exhibited no clear preference in either judgment.

All other cells in Table 2 (i.e., those that are not in bold) represent a non-inverse pattern of judgment. Some participants exhibited a thematic preference in both similarity and difference, while others exhibited a taxonomic preference in both judgments. Such occurrence of non-inversion within individual participants was nearly three times as common among Thematic participants (14.55\%) as among Taxonomic participants (5.45\%). Overall, 31\% of participants exhibited non-inverse patterns of judgment in the similarity and difference tasks. 
This result is even more powerful than Experiment 1a, since here the non-inversion occurred with the same participants judging the similarity and difference of the same items.

\section{Discussion}

In Experiment 2a (as in Experiment 1a) some participants tended to choose the taxonomic match as more similar and the thematic match as more different. Other participants demonstrated the opposite pattern (Figure 3), as predicted by the individualized model. Few participants behaved as though they had no preference for a particular type of match. Importantly, the Thematic participants did not represent a small group of outliers, as one might expect if the generalized model were correct. Rather, there were comparable numbers of Taxonomic and Thematic participants. The results of the present experiment also provide strong evidence that the non-inversion (Figure 4) is due to a differential influence of thematic relations on similarity and difference. Participants who exhibited the non-inversion were approximately three times more likely to prefer thematic choices than taxonomic choices (see Table 2). Because there were no order effects, the attenuation of thematic responding in comparison to Experiments 1a and $1 \mathrm{~b}$ was most likely the result of sampling differences. The individualized model predicts systematic between-participant disagreement, but does not specify the absolute dominance of thematic or taxonomic responding. Regardless of the overall number of Thematic participants in a sample, non-inversion is more likely to emerge from their judgments than from the judgments of Taxonomic participants.

\section{Experiment $2 b$}

Strikingly, some individuals in Experiment 2a systematically selected the same concept (typically the thematic match) as both more similar to and more different from the base concept. This somewhat surprising result raises the question of whether participants (a) intended to violate the inversion of similarity and difference, or (b) sought to uphold the inversion of similarity and difference, but failed to remember their similarity choices when 
providing their difference choices (or vice versa). To test these possibilities, in Experiment $2 \mathrm{~b}$ each participant judged both the similarity and the difference of the same triads on consecutive trials.

Method

Thirty-two undergraduates participated. The materials and procedure were the same as Experiment 2a except that participants judged the similarity and difference of each triad on consecutive trials. Order of presentation (similarity first or difference first) was randomized within-participants, so that each participant judged similarity first on half the trials and judged difference first on the other half. The order of item presentation was counterbalanced across two lists.

Results

Agreement. To test for an effect of presentation order (similarity first or difference first) we conducted a 2 (list) by 2 (judgment) ANOVA on thematic proportions, with participants random. Because the effect of list $[\mathrm{F}(1,30)=.05, \mathrm{p}=.82]$ and the list by judgment interaction $[\mathrm{F}(1,30)=.80, \mathrm{p}=.38]$ were not significant, we collapsed across order in the remaining analyses.

Despite judging similarity and difference on consecutive trials, participants continued to disagree in their choices (see Table 1 and Figure 5). Over half (62\%) of participants reliably selected the taxonomic match in similarity (Taxonomic participants) and a quarter (25\%) almost always chose the thematic match (Thematic participants). Only $13 \%$ of the participants had no clear preference for either match (Mixed participants). The pattern was the mirror image in difference, where most participants selected the thematic match (62\%) and a substantial minority selected the taxonomic match (25\%). Once again, few participants (13\%) exhibited no preference. As in the previous experiments, the distribution of similarity and difference judgments was characterized by between-participant disagreement. 
Non-inversion. The proportions of thematic choices in similarity and difference are plotted in Figure 6. Whereas in the previous experiments similarity and difference were moderately correlated, in this experiment the correlation was extremely strong $[\mathrm{r}(30)=-.99$, $\mathrm{p}<.001]$. Unlike the results of the previous experiments, neither type of option dominated similarity $[\mathrm{M}=.48, \mathrm{SE}=.03 ; \mathrm{t}(29)=-.57, \mathrm{p}=.57]$ or difference judgments $[\mathrm{M}=.51, \mathrm{SE}=$ $.03 ; \mathrm{t}(29)=.29, \mathrm{p}=.78]$. Thus, the overall thematic proportion did not differ from $.50[\mathrm{M}=$ $.49, \mathrm{SE}=.02 ; \mathrm{t}(29)=-1.47, \mathrm{p}=.15]$, thereby indicating an inverse relationship between similarity and difference judgments. In terms of response groups, every Thematic participant in similarity became a Taxonomic participant in difference, and vice versa; and every Mixed participant remained in that category across judgments (see Table 3). When judging similarity and difference on consecutive trials, every single participant (in terms of response groups) exhibited a complementary relationship between similarity and difference.

\section{Discussion}

The results of Experiment $2 \mathrm{~b}$ demonstrate that individual differences in similarity and difference judgments are robust even when these choices are made on consecutive trials for the same item (Figure 5). Although there was somewhat less thematic responding in similarity than in the previous experiments, a substantial proportion of participants remained resolutely Thematic. Participants in Experiment $2 \mathrm{~b}$ also tended to treat similarity and difference judgments as inverse (Figure 6). While almost a third (31\%) of participants in Experiment 2a departed from complementarity, none did so in Experiment $2 b$ (Table 3). Evidently, when memory allows, participants prefer to treat similarity and difference as inverses. Thus, noninversion is more than a group level phenomenon (Experiment 2a), but it appears to be sensitive to the proximity between similarity and difference judgments. 
Several indirect lines of evidence suggest that thematic integration is less likely to influence similarity judgments when participants are induced to process the stimuli more deeply. Gentner and Brem (1999) demonstrated that time-pressure influences the extent of thematic responding. They contrasted responses to triads with taxonomic and thematic matches to a base with responses to triads with taxonomic and unrelated matches. Participants were told to choose the taxonomically similar alternative and were given either $1000 \mathrm{~ms}$ or $2000 \mathrm{~ms}$ to respond. Participants incorrectly selected thematic matches more frequently than unrelated matches, suggesting that the thematic relatedness was confused with taxonomic similarity. Furthermore this effect was most prominent at the shorter deadline. Simmons and Estes (2008) found that instructing participants to base their judgments on features, and asking them to justify their responses, significantly attenuated the influence of thematic relations. And in the present experiments, we have found that thematic choices were less likely when similarity and difference judgments were directly contrasted on consecutive trials (Experiment 2b) than when the judgments occurred at different times (Experiment 2a). Given these findings, one could infer that thematic participants simply aren't very thoughtful; when induced to invest more thought into their judgments, thematic responding decreases. This inference is consistent with Gentner and Brem's (1999) suggestion that the thematic influence on similarity arises from inadvertently combining the outputs of comparison and integration.

The primary purpose of Experiment 3 was to test this hypothesis that thematic respondents are perhaps less thoughtful than their taxonomic peers. To investigate this possibility, we assessed participants' need for cognition (henceforth "NFC"), which is "the tendency for an individual to engage in and enjoy thinking" (Caccioppo \& Petty, 1982, p. 116). Given that individuals who are high in NFC tend to process information deeply (Lassiter, Briggs, \& Slaw, 1991; Levin, Huneke, \& Jasper, 2000), the hypothesis is that those same individuals should be less likely to make thematic judgments. Individuals low in NFC, 
in contrast, should be more likely to make thematic judgments. Thus, NFC should correlate negatively with thematic responding.

Alternatively, the thematic responding in the previous experiments could reflect participants' genuine belief that thematic relations are a legitimate component of similarity. That is, perhaps thematic participants and taxonomic participants simply possess different conceptions of similarity. To assess this explanation of thematic responding, we directly probed participants' beliefs about similarity. In particular, we gauged the extent to which participants agreed (or disagreed) that two things are similar if (a) they have the same features, (b) they belong in the same category, and (c) they occur in the same scenario. The first two potential sources of similarity (i.e., feature overlap and category co-membership) correspond to the comparison process and were expected to be favored by taxonomic respondents. The latter potential source of similarity (i.e., scenario co-occurrence) corresponds instead to thematic integration. The question of most interest here is whether thematic participants endorse scenario co-occurrence as a legitimate source of similarity. If so, that would indicate that their thematic responding is no mistake or confusion; thematic responding would be deliberate rather than thoughtless. If thematic participants were to reject scenario co-occurrence, however, this would support the supposition that thematic responding is inadvertent. So to summarize, if thoughtlessness explains thematic responding, then NFC should predict thematic preference. If, however, thematic responding reflects a belief about the nature of similarity, then participants' tendency to identify thematic relations as relevant to similarity should predict thematic preference.

A secondary purpose of Experiment 3 was to generalize the observation of thematic responding to more controlled stimuli and a potentially less biased task. Regarding the stimuli, the prevalence of thematic responding in the previous experiments could be due to various lexical factors such as word frequency, association, or co-occurrence frequency. That 
is, participants might have been inclined simply to choose the alternative that was more frequent, more strongly associated with the base, or more likely to co-occur with the base in ordinary language use. To address these possibilities, in Experiment 3 we developed a new set of stimuli for which the taxonomic and thematic alternatives were matched for word frequency, strength of association with the base concept, and frequency of co-occurrence with the base concept. Regarding the task, it is possible that the presentation of two options on each trial of the experiment could artificially inflate the prevalence of thematic responding; participants may infer an implicit demand to choose the thematic option on at least some of the trials. To address this concern, in Experiment 3 we used a rating task in which participants rated the similarity of the base concept (e.g., "cake") to a single other concept (e.g., "cookie"). To examine participants' preferences for taxonomic or thematic responding, we can compare for each base concept its similarity to the taxonomic alternative and its similarity to the thematic alternative. If an individual consistently rates the base concepts more similar to their thematic match than to their taxonomic match, then she would be a thematic participant. This methodology presumably will provide a less biased measure of the prevalence of thematic responding.

\section{Method}

Participants. Thirty-five undergraduates participated.

Materials \& Procedure. Items consisted of thirty base concepts, each paired with one taxonomic and one thematic alternative. For example, dungeon was paired with basement (taxonomic) and torture (thematic). Stimulus characteristics are summarized in Table 4, and a complete list of stimuli is presented in Table 5. As shown in Table 4, the taxonomic and thematic alternatives were matched for word frequency $(p=.55)$, forward association with the base $(p=.78)$, backward association with the base $(p=.47)$, and co-occurrence as indexed by both Latent Semantic Analysis (LSA; Landauer \& Dumais, 2002; $p=.60$ ) and Google hits ( $p$ 
$=.53)$. Thus, any difference in ratings of the taxonomic and thematic alternatives cannot be attributed to the lexical factors of frequency, association, or co-occurrence. To disguise the nature of the task, each base was also paired with an unrelated filler item. Items were organized into three blocked lists to counterbalance the order in which participants encountered the three alternatives to each base concept.

Participants were randomly assigned to one experimental list that included all 90 concept pairs (60 experimental and 30 filler). Each trial consisted of a base concept paired with a taxonomic match, thematic match, or unrelated filler, centrally presented on a computer display. Participants were instructed to rate the similarity, on a scale from 1 (not at all similar) to 7 (very similar) of the concept pairs. Responses were entered on the keyboard number pad. Items appeared in a pseudo-random order with the restriction that base concepts appeared only once in each third of a list, and with the order of taxonomic, thematic, and unrelated matches for each base counterbalanced across lists.

After the rating task participants completed the short form of the Need for Cognition scale (Cacciopo, Petty, \& Kao, 1984). Participants rated the extent of their agreement, on a scale from 1 (very strongly disagree) to 9 (very strongly agree), with 18 statements such as "I would prefer complex to simple problems" and "I find satisfaction in deliberating hard and for long hours.” Nine items (e.g., "Thinking is not my idea of fun.”) were negatively worded.

Finally, participants indicated their degree of agreement, on a scale from 1 (very strongly disagree) to 9 (very strongly agree) with the following statements, which were presented in random order:

Two things are similar if they have the same features.

Two things are similar if they belong in the same category.

Two things are similar if they occur in the same scenario.

\section{Results}


To test for an effect of list we conducted a 3 (list) by 3 (item type) ANOVA on similarity ratings with participants random. Because neither the main effect of list $[\mathrm{F}(2,32)=$ $1.79, \mathrm{p}=.18]$ nor the list by item type interaction $[\mathrm{F}(4,64)=.99, \mathrm{p}=.42]$ was significant, we collapsed across lists for the remaining analyses.

Agreement. Next, we calculated thematic preference based on the proportion of trials on which a participant assigned a higher rating to a thematic match with a base than to a taxonomic match with that same base. For example, a participant's rating for dungeon / torture was compared to his rating for dungeon / basement. A thematic response was recorded if the rating was higher for the thematic pair. A taxonomic response was recorded if the rating was higher for the taxonomic pair. Instances where a participant assigned the same rating to both pairs were excluded from analysis. Finally, we divided each participant's total thematic responses by the sum of thematic and taxonomic responses. This measure of thematic proportion ranged across participants from .00 to $.91(\mathrm{M}=.43, \mathrm{SE}=.05)$, indicating that substantial thematic responding was observed in the similarity ratings task, just as it was in the triad choice task of the preceding experiments. Importantly, the prevalence of thematic responding generalizes across tasks.

Participants were subsequently assigned to response groups on the basis of binomial probability. Because the number of relevant trials varied across participants (depending on how many taxonomic and thematic pairs elicited the same rating), binomial probability was assessed separately for each participant. As in the previous experiments, between-participant disagreement in thematic preference was evident (see Table 1): 40\% of participants were Taxonomic and $23 \%$ were Thematic. Thirty-seven percent of participants fell into the Mixed response group. Thus, individual differences in thematic responding were obtained even when word frequency, association strength, and co-occurrence frequency were controlled. To illustrate the discrepant ratings of these participant groups, Figure 7 shows the mean similarity 
ratings of taxonomic and thematic pairs by the Taxonomic, Mixed, and Thematic participants. As evident in the figure, Taxonomic participants rated the base concepts more similar to their taxonomic match than to their thematic match $[\mathrm{t}(11)=5.65, \mathrm{p}<.01]$, whereas Thematic participants rated the thematic matches more similar than the taxonomic matches $[t(7)=-$ $16.08, \mathrm{p}<.01]$. The Mixed participants rated the taxonomic and thematic pairs equally $(\mathrm{p}=$ .14). This pattern of mean similarity ratings validates the separation of participants into these three groups. It also highlights just how different the Taxonomic and the Thematic participants are in their perceptions of similarity.

Need for Cognition. NFC scores were based on participants' average rating on the questionnaire, with negatively worded items reverse-scored. Therefore, scores could theoretically range from 1 to 9 , with 1 representing low NFC and 9 representing high NFC. Participants exhibited a range of scores $(2.72-7.28)$ with a mean slightly above the midpoint $(\mathrm{M}=5.23, \mathrm{SE}=.19)$.

Beliefs about Similarity. Scores on the follow-up questions could range from 1 to 9 , with 1 indicating low agreement and 9 indicating high agreement. Participants exhibited a range of beliefs concerning the relevance of category co-membership (2-9), feature overlap $(2-9)$, and scenario co-occurrence $(1-9)$ to similarity. Category co-membership $(\mathrm{M}=7.06$, $\mathrm{SE}=.24)$ and feature overlap $(\mathrm{M}=6.91, \mathrm{SE}=.31)$ were perceived to be equally important to similarity $(\mathrm{p}=.49)$. However, scenario co-occurrence $(\mathrm{M}=4.91, \mathrm{SE}=.41)$ was perceived to be somewhat less important than either category co-membership $[\mathrm{t}(34)=5.70, \mathrm{p}<.001]$ or feature overlap $[\mathrm{t}(34)=4.74, \mathrm{p}<.001]$. Inter-correlations between these measures are reported in Table 6. Category co-membership was significantly positively correlated with feature overlap. Participants who thought that membership in the same category was important to similarity also thought that feature overlap was important. Category comembership was also positively related to scenario co-occurrence. Participants' follow-up 
question ratings were then correlated with their NFC scores (see Table 6). NFC was not associated with participants' beliefs about the importance of sharing features, belonging to the same category, or participating in the same scenario to similarity (all $p>.40$ ). The lack of association between NFC and scenario relevance was of interest. Evidently, participants who are low in NFC are no more likely than participants high in NFC to believe that scenario cooccurrence is relevant to similarity. Thus, if low NFC participants are indeed more likely to exhibit thematic responding, it would not be attributable to a belief that scenario cooccurrence is relevant to similarity.

Predicting Ratings. We initially tested whether participants' NFC, category relevance, feature relevance, and scenario relevance were correlated with their thematic proportion. As shown in Table 6, NFC and scenario relevance were both marginally associated with thematic preference $(\mathrm{p}=.08$ and $\mathrm{p}=.06$, respectively). We therefore ran a regression analysis with these two factors as predictors of thematic proportion. The overall model fit was significant [F $\left.(2,32)=4.58, \mathrm{p}<.05 ; \mathrm{R}^{2}=.22\right]$, with both $\mathrm{NFC}[\beta=-.35, \mathrm{p}<.05]$ and scenario relevance $[\beta$ $=.36, \mathrm{p}<.05]$ significantly predicting thematic proportion. Participants who reported a low need for cognition were more likely to rate thematic matches more similar than taxonomic matches. This finding supports the hypothesis that thematic responding may not require as much "deep" processing as taxonomic responding. However, those participants who exhibited greater thematic responding were also more likely to endorse scenario co-occurrence as a legitimate source of similarity. This latter finding suggests that some participants do believe that similarity should include thematic relations, and their ratings were consistent with this belief.

\section{Discussion}

Experiment 3 provides strong evidence for between-participant disagreement in the use of thematic relations in similarity judgments and demonstrates an effect of thematic 
relations using a novel, well-controlled item set. Therefore, the previous results cannot be attributed to lexical factors (i.e., word frequency, association strength, or co-occurrence) or to the use of a biasing task. Notably, there was considerable agreement among participants concerning the similarity of taxonomic items (Figure 7). All three participant types gave roughly equal ratings to taxonomic items. Thus, thematic preference in the triad task does not appear to indicate a rejection of taxonomic similarity. Rather, it indicates the importance of thematic relations relative to a taxonomic standard. For instance, among Taxonomic participants, thematic relations are relatively less important than taxonomic relations, while among Thematic participants, thematic relations are relatively more important than taxonomic relations.

The weighting of thematic relations in similarity judgments reflected differences in NFC. Participants who exhibited a strong thematic preference tended to enjoy thinking and problem solving less than participants who exhibited a weak thematic preference. The weighting of thematic relations was also associated with an overt belief that participation in a common scenario is a legitimate source of similarity. Participants with a strong thematic preference tended to rate thematic relations as more relevant to similarity than participants with a weak thematic preference. Interestingly, the observation that NFC and scenario cooccurrence themselves were uncorrelated suggests that there may be two types of the thematic participants - those who believe that thematic integration should be a component of similarity, and those who simply do not process the stimuli very deeply.

\section{General Discussion}

The preceding experiments have yielded three important results. First, these experiments revealed considerable individual differences in the perception of similarity and difference. Participants tended to choose the same type of stimulus across trials for a given judgment. In similarity judgments, for instance, some participants reliably chose the thematic 
match, while other participants reliably chose the taxonomic match. Few participants were agnostic in the choice between thematic and taxonomic stimuli. The surprising prevalence of thematic responding was equally evident whether judging which alternative is more "similar to" the base (Experiment 1a) or which is more "like" the base (Experiment 1b). In fact, participants continued to exhibit thematic similarity choices even when those judgments were directly contrasted with difference judgments of the same stimuli (Experiment 2a), though this thematic tendency was reduced when similarity and difference were judged on consecutive trials (Experiment 2b). Moreover, consistent thematic responding was evident not only in a triad choice task, but also in a similarity rating task (Experiment 3). Finally, participants' thematic tendencies were predicted by both their need for cognition and their beliefs about similarity. Specifically, participants with a high need for cognition were less likely to exhibit thematic responding, whereas participants who believe that scenario cooccurrence is a legitimate source of similarity were more likely to exhibit thematic responding. Although preliminary evidence supported the hypothesis of substantial individual variability in perceived similarity (Gentner \& Brem, 1999; Simmons \& Estes, 2008), these experiments provide the first demonstration of how robust this individual variability is, and how it relates to perceived difference.

The second important observation of these experiments is that thematic responding is not simply a by-product of lexical factors. Previous demonstrations of a thematic effect on similarity (Bassok \& Medin, 1997; Estes, 2003; Jones \& Love, 2007; Simmons \& Estes, 2008; Wisniewski \& Bassok, 1999) have not matched taxonomic and thematic items for potential confounds such as word frequency, association strength, and co-occurrence frequency. This is particularly problematic since many common thematically related concepts are also highly associated (e.g., milk / cow). However, a substantial number of participants in Experiment 3 rated thematic items as more similar than taxonomic items, even though these 
were matched for frequency, association, and co-occurrence. Thus, the thematic effect on similarity is not attributable to these lexical factors.

The third contribution of these experiments is to show that thematic relations may induce a non-inversion of similarity and difference. Experiment 1a corroborated the thematic non-inversion reported by Simmons and Estes (2008), and Experiment 2a provided the first within-participant demonstration of this non-inversion. Moreover, when participants' similarity and difference choices were non-inverse, it was most often the thematic option that was chosen as both more similar to and more different from the base. Notably, however, the non-inversion was eliminated entirely when participants judged both the similarity and the difference of the same items on consecutive trials (Experiment $2 b$ ). Thus, non-inversion is more than a group level phenomenon, but it is sensitive to the influence of participants' beliefs concerning similarity and difference. Below we discuss the theoretical and practical implications of these results.

These results clearly support a dual process model of perceived similarity. Single process models such as the contrast model (Tversky, 1977) and the structural alignment model (e.g., Gentner \& A. B. Markman, 1997) cannot explain the present results. If similarity was the result of comparison only, then participants should have chosen the taxonomic option consistently across trials, because taxonomically related stimuli are more easily aligned and have more commonalities than do taxonomically unrelated stimuli (A. B. Markman \& Gentner, 1993-a). So given the reliable and systematic occurrence of thematic choices, these results undermine comparison-only models of similarity. Simply stated, a model of similarity that does not account explicitly for thematic integration cannot explain participants' similarity choices.

These findings also indicate that the current, generalized version of the dual process model (Bassok \& Medin, 1997; Estes, 2003; Wisniewski \& Bassok, 1999) must be extended 
to describe accurately the influence of thematic integration on the perception of similarity. Understanding the systematic variability in thematic preference observed in the present experiments necessitates an individualized approach. In this model the weights assigned to the comparison and integration processes vary dramatically across individuals, but are consistent within an individual. Some participants weight comparison more than integration (e.g., Taxonomic participants), while others show the reverse pattern (e.g., Thematic participants). And, some participants assign the processes roughly equal weight (e.g., Mixed participants). Thus, the tendency to compare or integrate is constrained not only by stimulus and task factors, as originally proposed by Wisniewski and Bassok (1999), but also by an individual's preference for a given process. In particular, the results of Experiment 3 suggest that a preference for thematic integration in similarity judgments may arise from two independent sources. As suggested by Gentner and Brem (1999), some thematic respondents simply don't process the stimuli very deeply. For these participants, thematic integration may be confused with feature comparison. However, other thematic respondents are relatively more thoughtful, and they believe that thematic relations are important for similarity. These participants appear to respond thematically as a matter of deliberate choice.

Note that an individualized dual process model can also accommodate individual differences in categorization. Recall that some participants tend to form thematic categories, while others prefer to categorize taxonomically (Lin \& Murphy 2001; Murphy, 2001). Participants who put more weight on thematic relations in similarity judgments may go on to categorize thematically, and likewise participants who base their similarity judgments on attributes may tend to form taxonomic categories. That is, individual differences in the perception of similarity may explain the differences in categorization found by Murphy (2001; Lin \& Murphy, 2001). 
Like perceived similarity, perceived difference also necessitates an individualized dual process model. Some participants consistently chose the thematic alternative as more different from the base, whereas other participants consistently chose the taxonomic alternative. Despite the comparable pattern of results for similarity and difference choices, however, it is not sufficient to model difference as the opposite of similarity. As illustrated by the occurrence of non-inversion, perceived similarity and perceived difference diverge in the weighting of the thematic integration process. Specifically, the influence of thematic integration was attenuated in the perception of difference. This result corroborates Simmons and Estes (2008), who found that thematic relations account for less variance in difference judgments than in similarity judgments.

Thus, it appears that different types of information are relevant to similarity and difference. Our results suggest that non-inversion is tied to the performance of participants who prefer thematic relations in similarity, since these participants tend to answer less consistently than those who prefer comparison. However, this explanation should be interpreted with caution. As seen in Experiment 2b, non-inversion depends on the contiguity between similarity and difference judgments. Presenting similarity and difference consecutively eliminates non-inversion, presumably because participants believe that these judgments should be complementary.

The individual differences observed in the present experiments, although substantial, do not necessarily reflect large variability in conceptual knowledge; participants who categorize thematically are aware of the logic behind taxonomic categorization, and vice versa (Smiley \& Brown, 1979). Likewise, participants in similarity tasks of the sort reported here likely are aware of both thematic and taxonomic information. This presumed knowledge of both thematic and taxonomic information opens the possibility that individuals may respond inconsistently across time. That is, if an individual holds both sources of information, there is 
some probability that he may weight thematic information more heavily on one occasion than on another. Indeed, such within-participant inconsistency has been observed in categorization (Barsalou, 1989), although not in terms of a taxonomic versus thematic preference. However, two results argue against dramatic inconsistency in similarity judgments. First, Experiment 3 demonstrated that the precise weights that individuals assign to the outputs of comparison and integration reflect differences in NFC; Thematic participants are less likely than their Taxonomic counterparts to enjoy thinking and problem-solving. Because NFC is stable over time, thematic responding should also remain relatively stable over time. Furthermore, Thematic and Taxonomic participants appear to weight comparison and integration differently because they hold different beliefs about what information is relevant to similarity. Participants who believe that participation in the same scenario is important to similarity tend to exhibit a strong thematic preference. Intuitively, it seems unlikely that participants' beliefs about similarity will change substantially across time, and hence their similarity judgments should also remain stable across time. However, more direct investigation of withinparticipants consistency in similarity judgments may be a fruitful direction for further development of the individualized dual process model. 


\section{References}

Barsalou, L. W. (1982). Context-independent and context-dependent information in concepts. Memory \& Cognition, 10, 82-93.

Barsalou, L. W. (1989). Intraconcept similarity and its implications for interconcept similarity. In S. Vosniadou \& A. Ortony (Eds.), Similarity and analogical reasoning (pp. 76-121). Cambridge: Cambridge University Press.

Bassok, M., \& Medin, D. L. (1997). Birds of a feather flock together: Similarity judgments with semantically rich stimuli. Journal of Memory and Language, 36, 311-336.

Beck, J. (1966). Effect of orientation and of shape similarity on perceptual grouping. Perception \& Psychophysics, 1, 300-302.

Cacioppo, J. T., \& Petty, R. E. (1982). The need for cognition. Journal of Personality and Social Psychology, 42, 116-131.

Cacioppo, J. T., Petty, R. E., \& Kao, C. F. (1984). The efficient assessment of need for cognition. Journal of Personality Assessment, 48, 306-307.

Estes, Z. (2003). A tale of two similarities: Comparison and integration in conceptual combination. Cognitive Science 27, 911-921.

Estes, Z. \& Hasson, U. (2004). The importance of being nonalignable: A critical test of the structural alignment theory of similarity. Journal of Experimental Psychology: Learning, Memory, and Cognition, 30, 1082-1092.

Gati, I. \& Tversky, A. (1984). Weighting common and distinctive features in conceptual and perceptual judgments. Cognitive Psychology, 16, 341-370.

Gentner, D. \& Brem, S. K. (1999). Is snow really similar to a shovel? Distinguishing similarity from thematic relatedness. Proceedings of the $21^{\text {st }}$ Annual Conference of the Cognitive Science Society (pp. 179-184). Hillsdale, NJ: Lawrence Earlbaum Associates. 
Gentner, D. \& Gunn, V. (2001). Structural alignment facilitates the noticing of differences. Memory \& Cognition, 29, 565-577.

Gentner, D. \& Markman, A. B. (1994). Structural alignment in comparison: No difference without similarity. Psychological Science, 5, 152-158.

Gentner, D. \& Markman, A. B. (1997). Structure mapping in analogy and similarity. American Psychologist, 52, 45-56.

Goldstone, R. L. (1994). The role of similarity in categorization: Providing a groundwork. Cognition, 52, 125-157.

Hampton, J. A., Estes, Z., \& Simmons, S. (2007) Metamorphosis: Essence, appearance and behavior in the categorization of natural kinds. Memory \& Cognition, 35, 1785-1800.

Jones, M. \& Love, B. (2007). Beyond common features: The role of roles in determining similarity. Cognitive Psychology, 55, 196-231.

Landauer, T. K. \& Dumais, S. T. (2002). A solution to Plato's problem: The latent semantic analysis theory of the acquisition, induction, and representation of knowledge. Psychological Review, 104, 211- 240.

Lassiter, G. D., Briggs, M. A., \& Slaw, R. D. (1991). Need for cognition, causal processing, and memory for behavior. Personality and Social Psychology Bulletin, 17, 694-699.

Levin, I. P., Huneke, M. E., \& Jasper, J. D. (2000). Information processing at successive stages of decision making: Need for cognition and inclusion-exclusion effects. Organizational Behavior and Human Decision Processes, 82, 171-193.

Lin, E. L. \& Murphy, G. L. (2001). Thematic relations in adults' concepts. Journal of Experimental Psychology: General, 130, 3-28.

Markman, A. B. \& Gentner, D. (1993a). Splitting the difference: A structural alignment view of similarity. Journal of Memory and Language, 32, 517-535. 
Markman, A. B. \& Gentner, D. (1993b). Structural alignment during similarity comparisons. Cognitive Psychology, 25, 431-467.

Markman, E. M. \& Hutchinson, J. E. (1984). Children's sensitivity to constraints on word meaning: Taxonomic versus thematic relations. Cognitive Psychology, 16, 1-27.

Medin, D. L., Goldstone, R. L., \& Gentner, D. (1990). Similarity involving attributes and relations: Judgments of similarity and difference are not inverses. Psychological Science, 1, 64-69.

Medin, D. L., Goldstone, R. L., \& Gentner, D. (1993). Respects for similarity. Psychological Review, 100, 254-278.

Murphy, G. L. (2001). Causes of taxonomic sorting by adults: A test of the thematic-totaxonomic shift. Psychonomic Bulletin \& Review, 8, 834-839.

Navarro, D. \& Lee, M. (2004). Common and distinctive features in stimulus similarity: A modified version of the contrast model. Psychonomic Bulletin \& Review, 11, 961-974.

Nelson, D. L., McEvoy, C. L., \& Schreiber, T. A. (1998). The University of South Florida word association, rhyme, and word fragment norms. http://www.usf.edu/FreeAssociation

Simmons, S. \& Estes, Z. (2008). Dual processes of comparison and integration in similarity and difference. Manuscript under review.

Smiley, S. S. \& Brown, A. L. (1979). Conceptual preference for thematic or taxonomic relations: A nonmonotonic age trend from preschool to old age. Journal of Experimental Child Psychology, 28, 249-257.

Tversky, A. (1977). Features of similarity. Psychological Review, 84, 327-352.

Wisniewski, E. J. \& Bassok, M. (1999). What makes a man similar to a tie? Stimulus compatibility with comparison and integration. Cognitive Psychology, 39, 208-238. 
Table 1. Descriptive statistics of participant groups (Taxonomic, Mixed, Thematic) as a function of task (Similarity, Difference), Experiments 1-

3.

Taxonomic

Mixed

\begin{tabular}{|c|c|c|c|c|c|c|c|c|c|c|c|c|c|c|c|c|}
\hline \multirow[b]{2}{*}{ Exp't } & \multirow[b]{2}{*}{ Task } & \multicolumn{5}{|c|}{ Taxonomic } & \multicolumn{5}{|c|}{ Mixed } & \multicolumn{5}{|c|}{ Ihema } \\
\hline & & $\mathrm{N}$ & Min & Max & $\mathrm{M}$ & $\mathrm{SE}$ & $\mathrm{N}$ & Min & Max & $\bar{M}$ & $\mathrm{SE}$ & $\mathrm{N}$ & Min & Max & $\mathrm{M}$ & $\mathrm{SE}$ \\
\hline \multirow[t]{2}{*}{$1 \mathrm{a}$} & Sim & 11 & 0.00 & 0.20 & 0.05 & 0.02 & 8 & 0.37 & 0.67 & 0.50 & 0.04 & 16 & 0.73 & 1.00 & 0.90 & 0.02 \\
\hline & Dif & 13 & 0.00 & 0.30 & 0.12 & 0.03 & 7 & 0.37 & 0.63 & 0.46 & 0.04 & 13 & 0.73 & 0.97 & 0.89 & 0.02 \\
\hline $1 \mathrm{~b}$ & Sim & 11 & 0.00 & 0.23 & 0.07 & 0.02 & 4 & 0.37 & 0.60 & 0.52 & 0.05 & 20 & 0.77 & 1.00 & 0.92 & 0.02 \\
\hline \multirow[t]{2}{*}{$2 \mathrm{a}$} & Sim & 30 & 0.00 & 0.30 & 0.09 & 0.02 & 4 & 0.33 & 0.67 & 0.51 & 0.07 & 21 & 0.70 & 1.00 & 0.89 & 0.02 \\
\hline & Dif & 12 & 0.00 & 0.27 & 0.08 & 0.02 & 9 & 0.37 & 0.60 & 0.50 & 0.03 & 34 & 0.70 & 1.00 & 0.91 & 0.02 \\
\hline \multirow[t]{2}{*}{$2 b$} & Sim & 20 & 0.00 & 0.20 & 0.08 & 0.02 & 4 & 0.33 & 0.43 & 0.38 & 0.03 & 8 & 0.83 & 1.00 & 0.91 & 0.02 \\
\hline & Dif & 8 & 0.00 & 0.17 & 0.09 & 0.02 & 4 & 0.57 & 0.67 & 0.63 & 0.02 & 20 & 0.73 & 1.00 & 0.88 & 0.02 \\
\hline 3 & Sim & 12 & 0.00 & 0.32 & 0.11 & 0.03 & 15 & 0.35 & 0.64 & 0.50 & 0.03 & 8 & 0.68 & 0.91 & 0.79 & 0.03 \\
\hline
\end{tabular}


Table 2. Distribution of participants' choice patterns (Taxonomic, Mixed, Thematic) in each task (Similarity, Difference), Experiment 2a.

\begin{tabular}{|c|c|c|c|c|c|}
\hline & \multicolumn{3}{|c|}{ Difference } & \multirow[b]{2}{*}{ Total } \\
\hline & & Taxonomic & Mixed & Thematic & \\
\hline \multirow{4}{*}{ Similarity } & Taxonomic & $5.45 \%$ & $3.64 \%$ & $45.45 \%$ & $54.55 \%$ \\
\hline & Mixed & $1.82 \%$ & $3.64 \%$ & $1.82 \%$ & $7.27 \%$ \\
\hline & Thematic & $20.00 \%$ & $3.64 \%$ & $14.55 \%$ & $38.19 \%$ \\
\hline & Total & $27.27 \%$ & $10.91 \%$ & $61.82 \%$ & \\
\hline
\end{tabular}


Table 3. Distribution of participants' choice patterns (Taxonomic, Mixed, Thematic) in each task (Similarity, Difference), Experiment 2b.

\begin{tabular}{|c|c|c|c|c|c|}
\hline & \multicolumn{3}{|c|}{ Difference } & \multirow{3}{*}{$\begin{array}{c}\text { Total } \\
62.00 \%\end{array}$} \\
\hline & & Taxonomic & Mixed & Thematic & \\
\hline \multirow{4}{*}{ Similarity } & Taxonomic & $0.00 \%$ & $0.00 \%$ & $62.00 \%$ & \\
\hline & Mixed & $0.00 \%$ & $13.00 \%$ & $0.00 \%$ & $13.00^{\circ}$ \\
\hline & Thematic & $25.00 \%$ & $0.00 \%$ & $0.00 \%$ & $25.00 \%$ \\
\hline & Total & $25.00 \%$ & $13.00 \%$ & $62.00 \%$ & \\
\hline
\end{tabular}


Table 4. Stimulus characteristics, Experiment 3.

\begin{tabular}{|c|c|c|c|c|c|c|c|c|c|c|}
\hline \multirow[b]{3}{*}{ Alternative } & & & \multicolumn{4}{|c|}{ Association } & \multicolumn{4}{|c|}{ Co-occurrence } \\
\hline & \multicolumn{2}{|c|}{ Word Frequency } & \multicolumn{2}{|c|}{ Forward } & \multicolumn{2}{|c|}{ Backward } & \multicolumn{2}{|c|}{ LSA } & \multicolumn{2}{|c|}{ Google } \\
\hline & $\mathrm{M}$ & SE & $\mathrm{M}$ & SE & $\mathrm{M}$ & SE & M & $\mathrm{SE}$ & $\mathrm{M}$ & SE \\
\hline Taxonomic & 23.8 & 6.6 & 0.15 & 0.04 & 0.10 & 0.04 & 0.39 & 0.03 & 9.25 & 4.26 \\
\hline Thematic & 29.5 & 6.6 & 0.16 & 0.04 & 0.14 & 0.03 & 0.36 & 0.04 & 6.35 & 2.25 \\
\hline
\end{tabular}

Note. "Word Frequency" = written frequency in the Kucera-Francis norms; "Forward Association" = probability of producing the base given the alternative, from the Nelson et al. (1998) norms; "Backward Association" = probability of producing the alternative given the base, from the Nelson et al. (1998) norms; "LSA" = cosine values from the "General reading up to $1^{\text {st }}$ year college" online corpus; "Google" = number of hits (in millions) obtained in a search of "[alternative] [base]" on google.com, data retrieved on 9/21/2007. The taxonomic and thematic alternatives did not differ significantly in any of these measures. 
Table 5. Stimuli, Experiment 3.

$\begin{array}{lll}\text { Base } & \text { Taxonomic } & \text { Thematic } \\ \text { bee } & \text { butterfly } & \\ \text { bird } & \text { robin } & \text { honey } \\ \text { cake } & \text { cookie } & \text { nest } \\ \text { carrot } & \text { celery } & \text { birthday } \\ \text { cow } & \text { pig } & \text { rabbit } \\ \text { crown } & \text { hat } & \text { ranch } \\ \text { dog } & \text { cat } & \text { queen } \\ \text { dungeon } & \text { basement } & \text { bone } \\ \text { dust } & \text { soot } & \text { torture } \\ \text { flower } & \text { plant } & \text { broom } \\ \text { fur } & \text { hair } & \text { vase } \\ \text { jar } & \text { bottle } & \text { coat } \\ \text { knight } & \text { soldier } & \text { jelly } \\ \text { lamp } & \text { flashlight } & \text { armor } \\ \text { monkey } & \text { primate } & \text { desk } \\ \text { movie } & \text { documentary } & \text { banana } \\ \text { needle } & \text { pin } & \text { producer } \\ \text { net } & \text { rope } & \text { thimble } \\ \text { oyster } & \text { clam } & \text { fish } \\ \text { pastry } & \text { tart } & \text { pearl } \\ \text { prison } & \text { jail } & \text { baker } \\ \text { river } & \text { lake } & \text { criminal } \\ \text { robe } & \text { cloak } & \text { boat } \\ \text { sapphire } & \text { emerald } & \text { bath } \\ \text { sheep } & \text { goat } & \text { ring } \\ \text { ship } & \text { yacht } & \text { wool } \\ \text { shirt } & \text { jacket } & \text { sailor } \\ \text { smoke } & \text { smog } & \text { tie } \\ \text { squirrel } & \text { rat } & \text { fire } \\ \text { tent } & \text { hut } & \text { nut } \\ & & \text { camp } \\ & & \\ \text { limo } & & \end{array}$


Table 6. Inter-correlations (r) between Thematic preference, NFC, and follow-up questions, Experiment 3.

\begin{tabular}{llll} 
Thematic & Category & Feature & Scenario \\
Preference & Relevance & Relevance & Relevance \\
\hline
\end{tabular}

Category

Relevance

.17

Feature

Relevance $.19 \quad .74 *$

Scenario

Relevance

$.42 *$

.32

NFC $\quad-.30^{\dagger}$
Note. ${ }^{*} \mathrm{p}<.05 ;{ }^{\dagger} \mathrm{p}<.08 . \mathrm{df}=35$

.11

.12 
Figure 1. Distribution of participants' choice patterns (Taxonomic, Mixed, Thematic) as a function of task (Similarity, Difference), Experiment 1a.

$\square$ Taxonomic $\square$ Mixed $\square$ Thematic

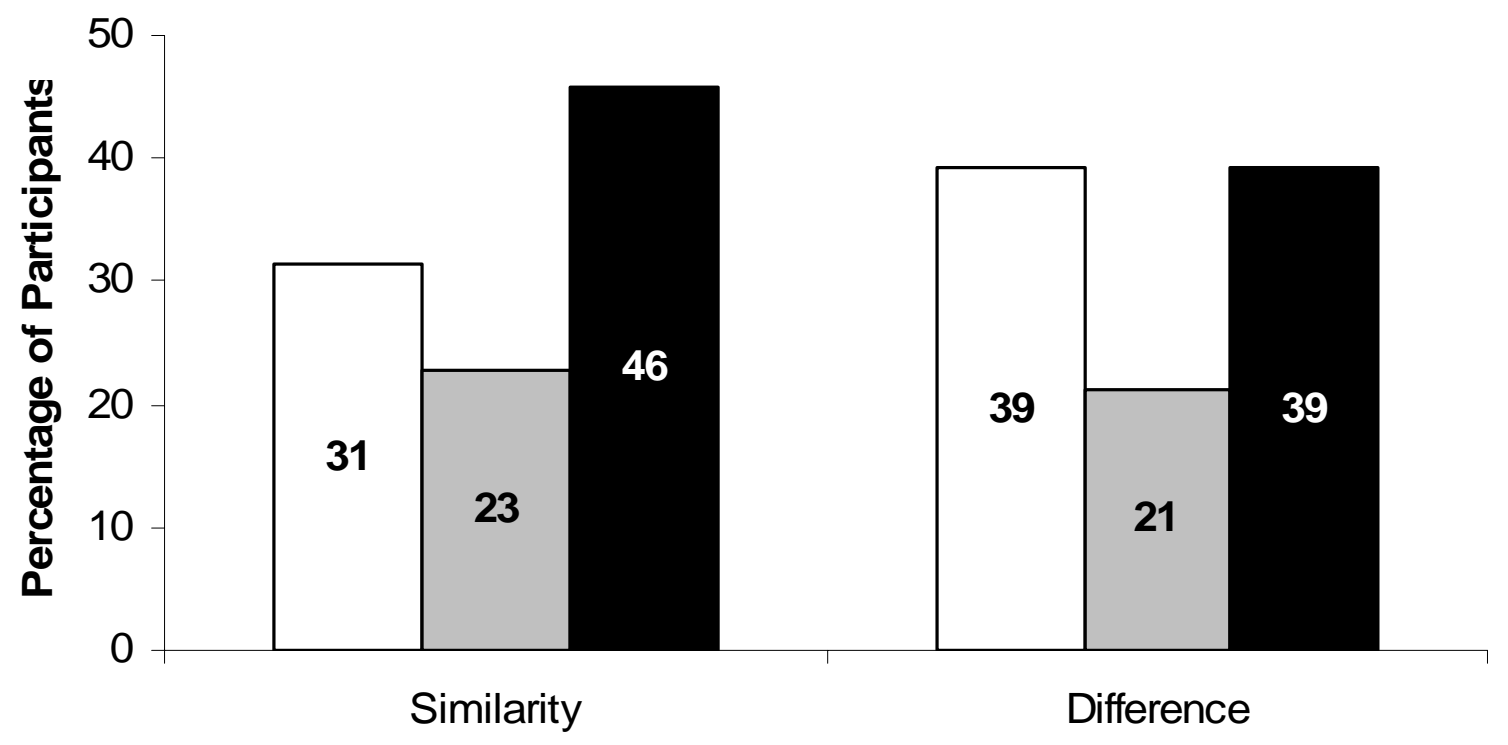


Figure 2. Proportion of thematic choices in difference plotted as a function of proportion of thematic choices in similarity, Experiment 1a.

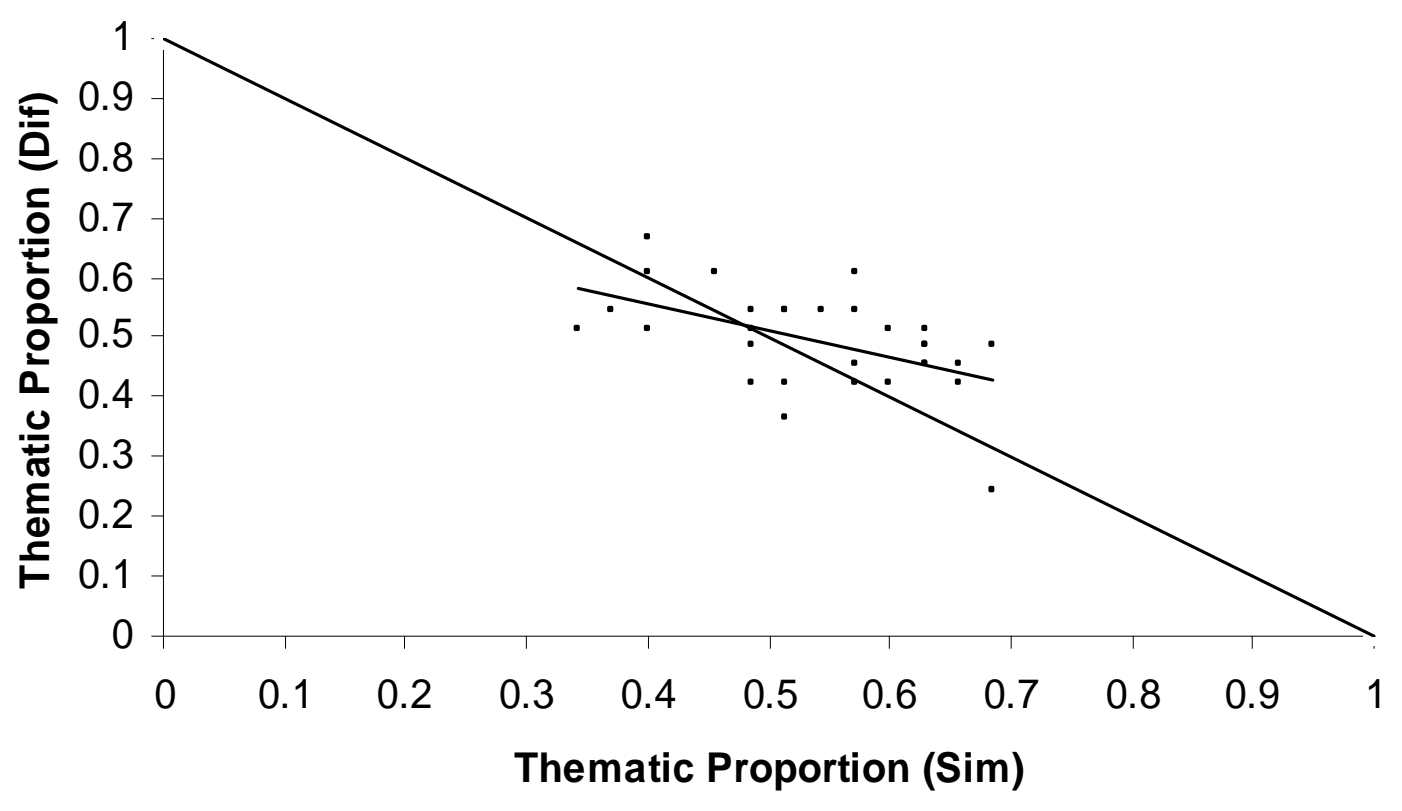


Figure 3. Distribution of participants' choice patterns (Taxonomic, Mixed, Thematic) as a function of task (Similarity, Difference), Experiment 2a.

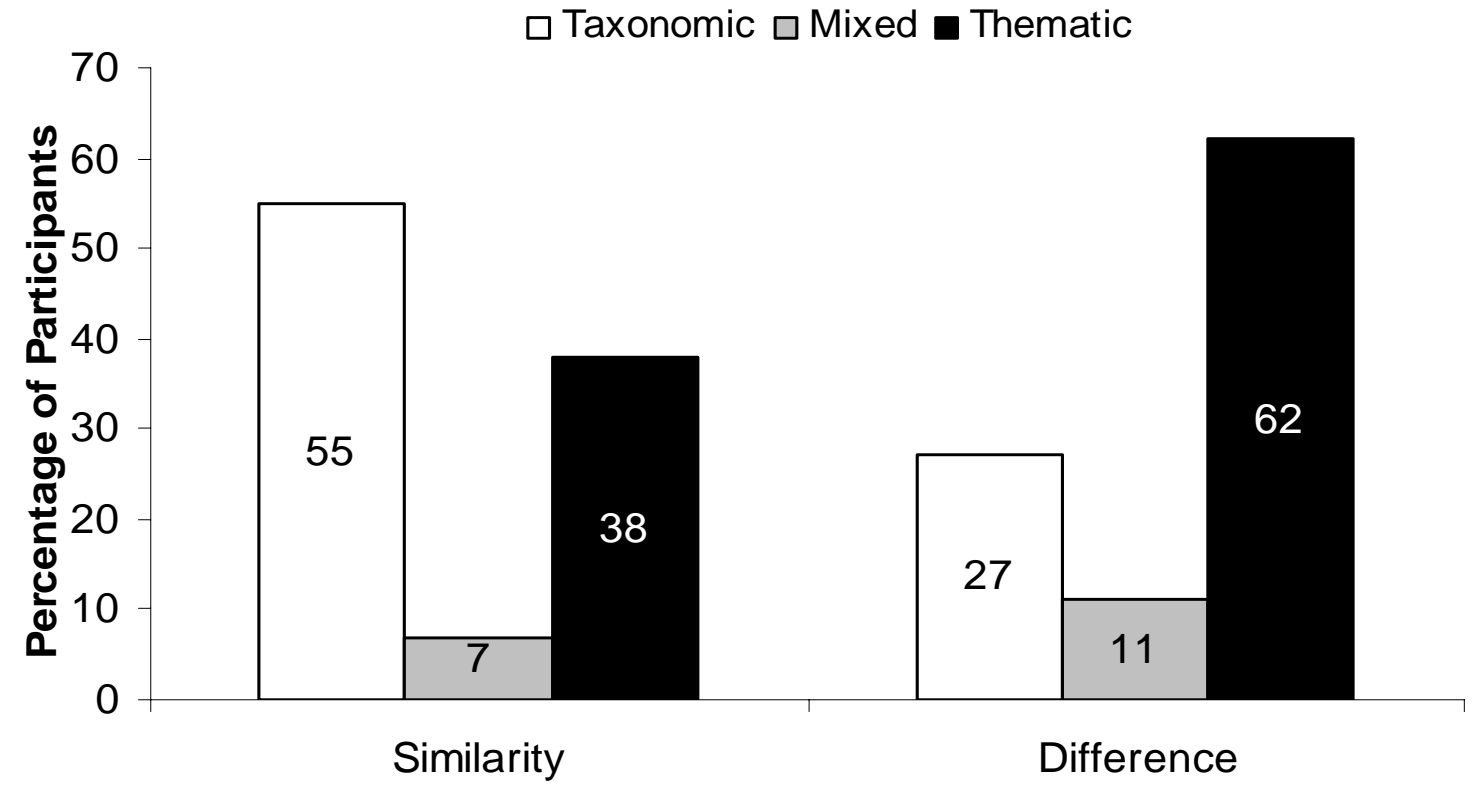


Figure 4. Proportion of thematic choices in difference plotted as a function of proportion of thematic choices in similarity, Experiment 2a.

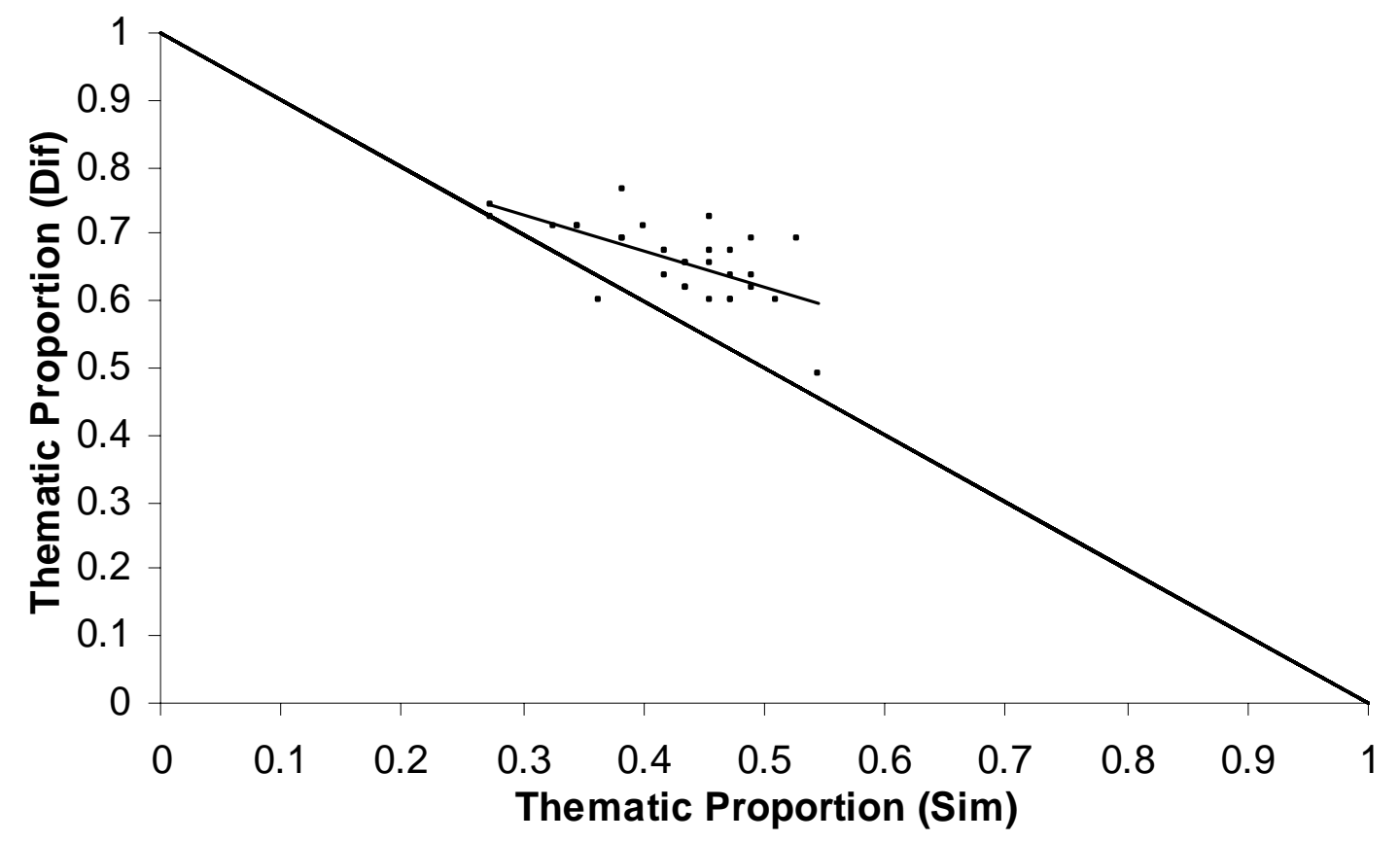


Figure 5. Distribution of participants' choice patterns (Taxonomic, Mixed, Thematic) as a function of task (Similarity, Difference), Experiment $2 b$.

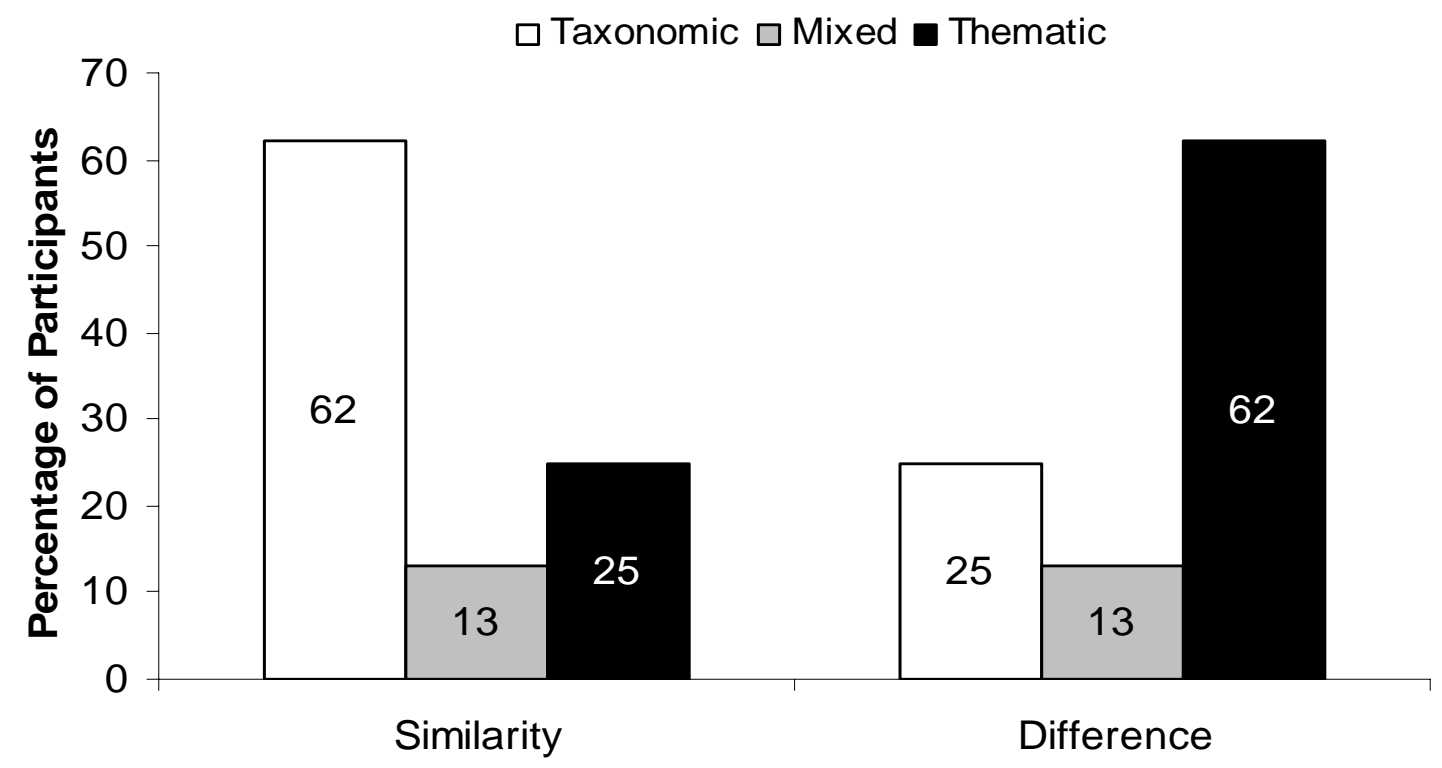


Figure 6. Proportion of thematic choices in difference plotted as a function of proportion of thematic choices in similarity, Experiment $2 b$.

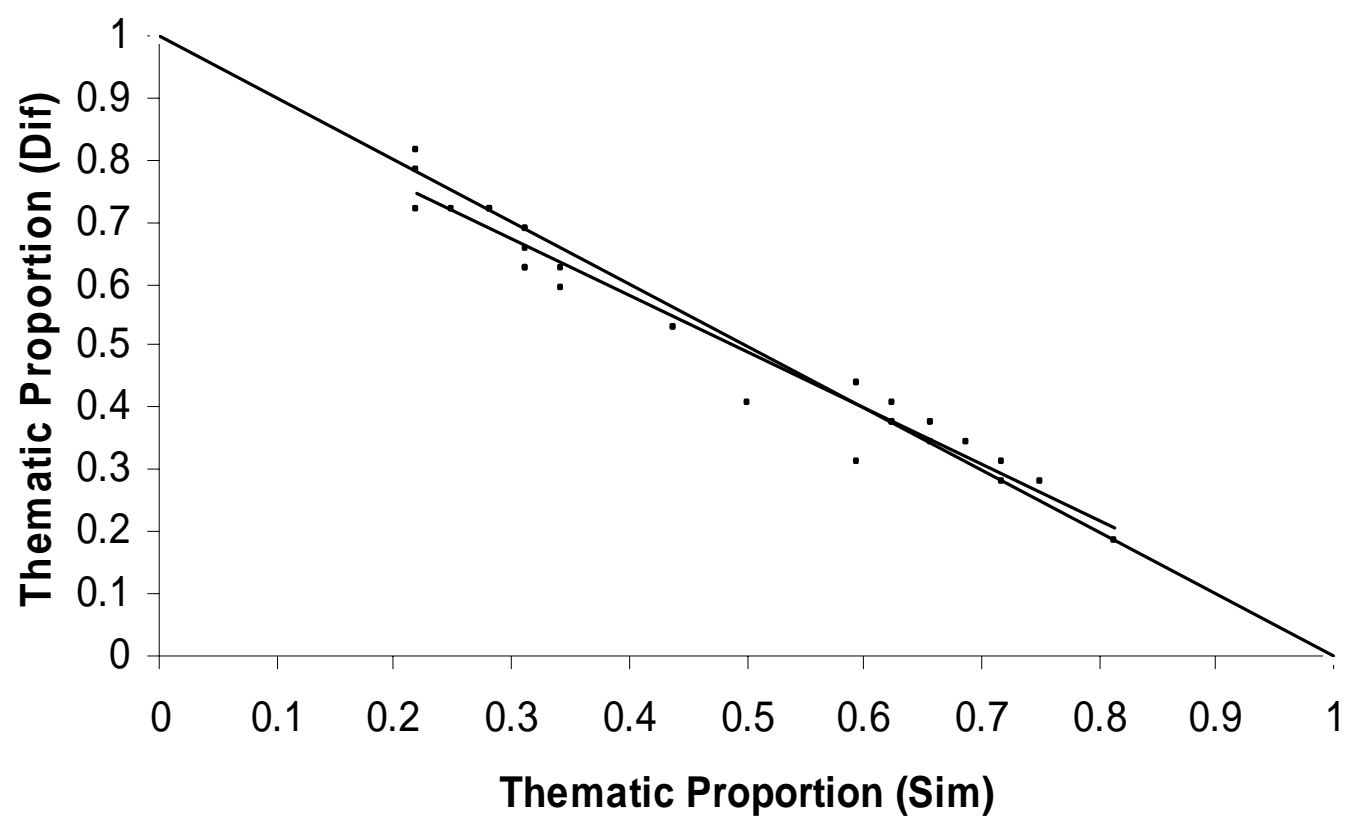


Figure 7. Mean similarity ratings as a function of response group and item type, Experiment 3.

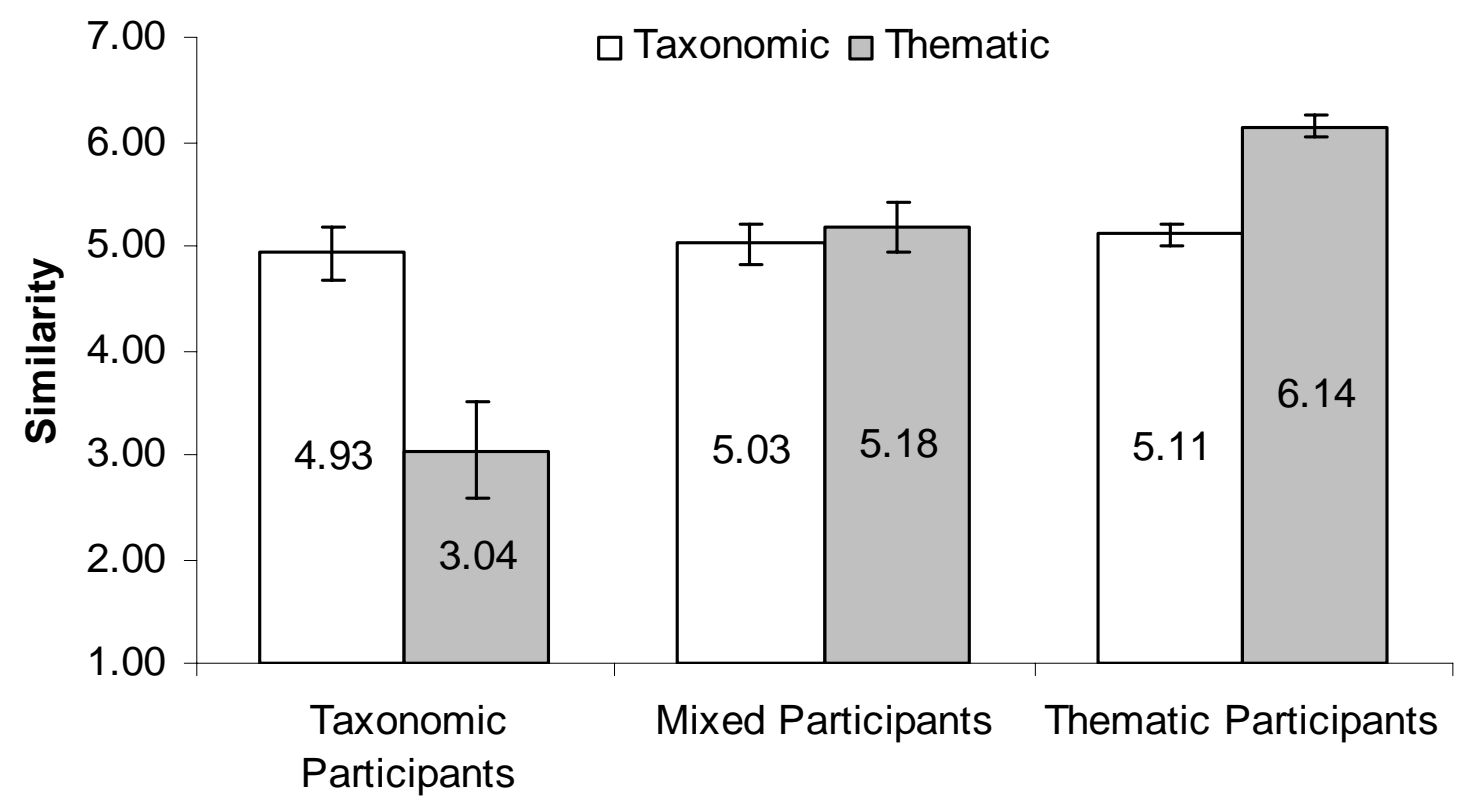

